\title{
Radiative Transfer and Dynamics of Stellar Outflows
}

\author{
NATHAN NETZER \\ OR'T Braude College, Karmiel, Israel
}

The equation of radiative transfer is solved for spherically symmetric outflows. This is done by expanding the intensity function into Legendre series. The radiative transfer is coupled with the equation of motion of the outflow, where the driving force is radiation pressure on dust. It is found that there is a correlation between the outflow velocity of the gas and the mass-loss rate. There is a maximum possible mass-loss rate for carbon stars, which is of the order of $10^{-4} M_{\odot} \mathrm{yr}^{-1}$. There is probably such a limit for oxygen stars as well, but it is much higher, as attempts to carry out the calculation to above $10^{-3} M_{\odot} \mathrm{yr}^{-1}$ do not show any decrease in their outflow velocity. 